\title{
The Firing Dispersion of Bullet Test Sample Analysis
}

\author{
Youliang Xu, Junbin Zhang, Li Ma, and Yonghai Shan
}

\begin{abstract}
For the past, bullet dispersion test firing bullets more than the number of groups, the consumption of large bombs and other issues, This article adopts a new method, using the previous tests produce a lot of dispersion test data, basing on statistical theory of bullets dispersion test sample size in-depth analysis and research to determine the test firing is reasonably necessary number of groups and single group number issued, which will help bullet-dispersion test methods to improve and perfect. Using this method, we can use the previous bullet shooting precision data, scientifically determine the bullet intensive experimental group number and the numbers each group. Save ammunition test fund and time.
\end{abstract}

Index Terms-Bullet, dispersion, test, the number of bullets.

\section{INTRODUCTION}

Weapons systems production and processing is a complex process, despite the strict control of the production conditions, there are individual differences, but also in the shooting, also cannot control the shooting condition and weather condition completely, so the ammunition in the placement appears scatter is inevitable. Because of effect of initial velocity, angle and the ballistic coefficient and the resulting dispersal distance; direction spread because of azimuth angle, initial disturbance and the bias current (drift) effect. So , the intensity of weapon system is affected by many uncertain factors, therefore, the intensity of result evaluation should not only rely on the small sample test data were randomly selected to, but should use the method of mathematical statistics to carry on the system analysis. The current domestic dispersion test [1], mainly has the following two kinds of test and evaluation methods:

\section{A. A Series of Test Method}

Tested under the condition of normal temperature is shooting 6 groups, 20 in each group, the mean value of the test results are compared with the tactical and technical index, and get the conclusion of the sample dispersion whether meet the requirements.

\section{B. The Method of Hypothesis Testing}

Statistical analysis of experimental data, using K2 test on the tested concentration whether meet the requirements given the exact conclusion.

At present, the range of dispersion test is taken by the first method, this method is based on the test data are averaged to eliminate the uncertainty of shooting and meteorological conditions of the resulting effects on the dispersion, the estimated value is closer to the true intensity of the test values.

Manuscript received October 9, 2014; revised December 25, 2014.

The authors are with the Baicheng Ordnance Test Center, China (e-mail: hemmler@163.com).
Undisputed, this approach does reduce the influence of random factors in a certain extent, but only decreases and has not been completely eliminated, effect still exists, but inversely with the amount of sample selection, the larger the sample size, the less effect, shooting range test data is more close to the true value, but the actual situation is not possible samples range test the extracted amount is large enough, so the estimate value does not exist can be compared with the true value. In this paper, statistical analysis, and strive to draw the permissible level of significance, and kept within the limits of pseudo-probability optimum firing.

\section{SCATtered Projectiles Relationship With $R_{50}$}

In China, the projectile dispersion models always use [2]:

$$
f(y, z)=\frac{1}{2 \pi \delta_{y} \delta_{z}} e^{-\frac{1}{2}}\left[\frac{\left(y-a_{y}\right)^{2}}{\delta_{y}^{2}}+\frac{\left(z-a_{z}\right)^{2}}{\delta_{z}^{2}}\right]
$$

The formula:

$f(y, z)$ : Height, direction of coordinate $(y, z)$ probability dispersion function;

$y, z$ : The probability dispersion function of the variables, namely the impact point coordinate;

$a_{y}, a_{z}$ : The level and direction of the coordinates of the center point of impact spread;

$\delta_{y}, \delta_{z}:$ The level and direction of the impact point spread standard deviation.

The fire intensity of $R_{50}$, on the basis of the definition of R50, the:

$$
\iint_{y^{2}+z^{2} \leq R_{50}^{2}} f(y, z) d y d x=0.5
$$

Put the $(y, z)$ of the probability dispersion function of $f(y, z)$ into type. There are:

$\iint_{y^{2}+z^{2} \leq R_{50}^{2}} \frac{1}{2 \pi \delta_{y} \delta_{z}} e^{-\frac{1}{2}\left[\frac{\left(y-a_{y}\right)^{2}}{\delta_{y}^{2}}+\frac{\left(z-a_{z}\right)^{2}}{\delta_{z}^{2}}\right]} d y d x=0.5$

Let $y=r \cos \theta, z=r \sin \theta ;(0 \leq \theta \leq 2 \pi)$,then: 
Assumption coincides of projectiles dispersion center and the center of the target or target point [3], there are $a_{y}=a_{z}=0$, formula (4) can be transformed into:

$$
\frac{1}{2 \pi \delta_{y} \delta_{z}} \int_{0}^{R_{50}} \int_{0}^{2 \pi} d \theta e^{-\frac{r^{2}}{2}\left[\frac{\cos \theta^{2}}{\delta_{y}^{2}}+\frac{\sin \theta^{2}}{\delta_{z}^{2}}\right]} r d r=0.5
$$

Hypothesis a projectile overall as two-dimensional normal random variable $y, z$, the standard deviation is:

$$
\begin{aligned}
& \hat{\delta}_{y}=\sqrt{\frac{\sum_{i=1}^{n}\left(y_{i}-\bar{y}\right)^{2}}{n-1}} \\
& \hat{\delta}_{z}=\sqrt{\frac{\sum_{i=1}^{n}\left(z_{i}-\bar{z}\right)^{2}}{n-1}}
\end{aligned}
$$

where $\hat{\delta}$ is the estimated value of standard deviation.

\section{THE SAMPLE SizE Estimation OF SHOOTING}

A. Given Significance Level and Kept Shooting Pseudo-Probability Sample of the Total Amount

To determine the sample size $n$, is one of the most important problems in the dispersion test. In the parameter estimation, there are two methods for determining sample size:

1) The accuracy of the estimates requested this requirement to determine the sample size

2) $\varepsilon$ permissible deviation is given as well as the confidence level $1-\alpha$, according to the given $\varepsilon$ and $1-\alpha$, determine the sample size. Given the probability of making the first mistake and allowed deviation $\varepsilon$, overall distribution in the $\hat{R}_{50}$ case it is unknown $S$ whether the comparative sample $R_{50}$ and $\hat{R}_{50}$ required overall $n$, the confidence level for a given $1-\alpha$, the average y means included in the interval $(\bar{X}-\varepsilon, \bar{X}+\varepsilon)[4]$.

In the current bullets intensity test, a design-dispersion requirement is $\left(R_{50}\right)_{0}$ and $R_{50}$ according to the given test when a significant level $\alpha$ decided to test the boundaries. When the intensity index exceeds the specified value to a certain extent $\left(R_{50}=\left(R_{50}\right)_{1}\right)$, the probability of its existence no more than pseudo- $\beta$ [5]. Do statistic $\chi^{2}$ :

$$
\chi^{2}=\frac{n-1}{\left(R_{50}\right)_{0}^{2}} R_{50}^{2}
$$

When the $R_{50}=\left(R_{50}\right)_{0}$ under the conditions established, the statistic degrees of freedom of the $\chi^{2}$ variable, for a given level of significance $\alpha$, can determine boundaries $\chi_{\alpha}^{2}$. So that $P\left(\chi^{2} \succ \chi_{\alpha}^{2}\right)=\alpha$.

When the $R_{50}=\left(R_{50}\right)_{0}$ under the conditions established by the probability of $1-\beta$, may decide to $\chi_{1-\beta}^{2}$, so that $P\left(\chi^{2} \succ \chi_{1-\beta}^{2}\right)=1-\beta$.

This has $\chi_{1-\beta}^{2}=\frac{1}{r^{2}} \chi_{\alpha}^{2}$, where $r=\frac{\left(R_{50}\right)_{1}}{\left(R_{50}\right)_{0}}$.

The upper side is given probability $P, \chi^{2}$ satisfy the threshold value $\chi_{P}^{2}, P=P\left(\chi^{2} \succ \chi_{P}^{2}\right)$, drawn:

$$
P=\int_{\sqrt{2} \chi_{P}^{2}}^{\infty} \frac{1}{\sqrt{2 \pi}} \mathrm{e}^{-\frac{(\mathrm{x}-\sqrt{2 \mathrm{v}-1})^{2}}{2}} d x
$$

It follows that the overall sample:

$$
v=\frac{1}{2}+\frac{1}{2}\left(\frac{u_{\alpha}+r u_{\beta}}{r-1}\right)^{2}
$$

The overall sample size can be expressed as

$$
n=\frac{3}{2}+\frac{1}{2}\left(\frac{u_{\alpha}+r u_{\beta}}{r-1}\right)^{2}
$$

\section{B. The Total Sample Size and Error Analysis}

For $R_{50}$, with formula (8), sample size can be calculated as:

$$
\frac{\varepsilon}{\left(R_{50}\right)_{0}}=\frac{\left(R_{50}\right)_{1}-\left(R_{50}\right)_{0}}{\left(R_{50}\right)_{0}}=(r-1)=\frac{u_{\alpha}+\sqrt{2 n-3}}{\sqrt{2 n-3}-u_{\beta}}-1
$$

The $\frac{\varepsilon}{\left(R_{50}\right)_{0}}$ is the relative test error.

From the above formula can be seen, with the increase of overall sample size, the relative errors are gradually reduced, when the sample size tends to infinity, the relative test error tends to zero.

The total sample above formula from zero to infinity [6], the calculated relative test error trend with the change of sample size as shown in Fig. 1.

As can be seen, the relative test error decreases as the sample size increased overall, but the sample size after more than one hundred rounds of ammunition, the relative test error decrease slowly. Can be selected in the test error conditions are relatively acceptable under economic sample volume.

In order to further study of the relative error changes with the sample size, the Fig. 1 amplification (see Fig. 2), it can be seen from Fig. 2, the test sample size of more than 40 rounds of ammunition, the relative error is less than 0.1 . (reducing test application percentage or scores of thousands). So, need 
to test meet the intensity overall sample volume dispersion check 40 bullets can be better.

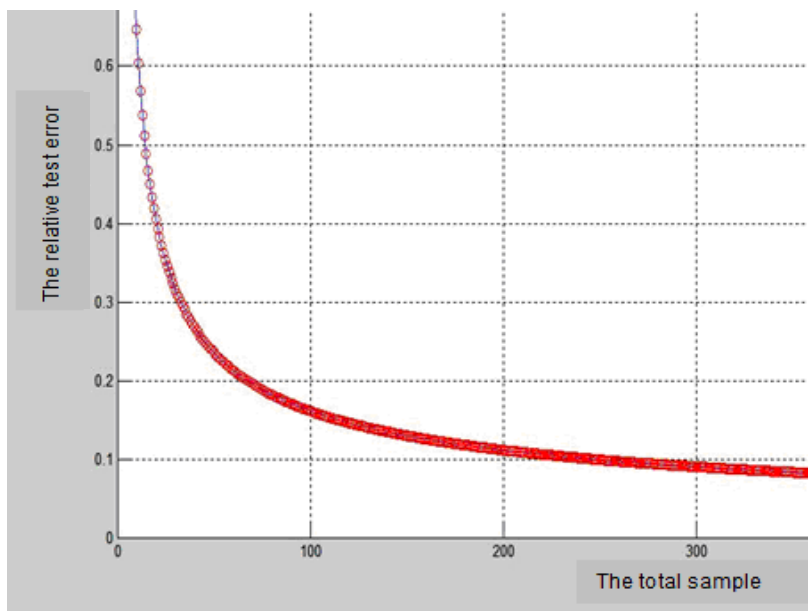

Fig. 1. Relative to examine the relationship between error and the overall sample size.

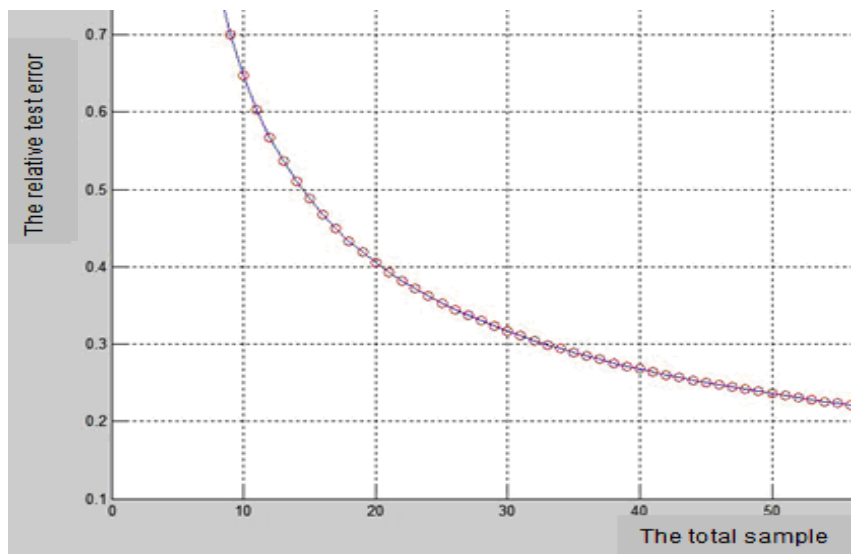

Fig. 2. Partial enlargement of Fig 1 .

That is: when the $\alpha=10 \%, \beta=20 \%$, the total sample shooting is $n=40$.

\section{Shooting Group Number and the Number of Its} Estimation Accuracy

Generally speaking, in the firing dispersion test, often some system errors, and interfere with test results. In order to reduce the interference, we often use multiple shooting methods.

According to the limit law, system error $\Delta$ random obey normal distribution, the distribution center for the $\mathrm{O}$, variance of $\mathrm{O}_{\Delta}$

In the shooting test group and each shot number the same, half will hit the radius estimation formula for $R_{50}$ :

$$
\hat{R}_{50}=\sqrt{\frac{1}{m} \sum_{i=1}^{m} \hat{R}_{50 i}^{2}}
$$

For the $R_{50}$, can be approximated as unbiased estimation, the estimation accuracy is:

$$
\delta_{\hat{R}_{50}}=\frac{R_{50}}{\sqrt{2 \sum_{i=1}^{m}(n-1)}}=\frac{R_{50}}{\sqrt{2 m(n-1)}}
$$

Assuming dispersion test, optimum sample size for $N=40$, i.e.: $m n=N$, It can consider two cases.

Fixed $n$, make $m$ change; or fixed $m, n$ changes.

That is: $m=\frac{40}{n}, n=\frac{40}{m}$

Will it into (9) formula, there is:

$$
\begin{gathered}
\delta_{\hat{R}_{50}}=\frac{R_{50}}{\sqrt{80\left(\frac{n-1}{n}\right)}} \\
\delta_{\hat{R}_{50}}=0.7071 \frac{R_{50}}{\sqrt{40-m}}
\end{gathered}
$$

The formula (11), (12), the number of groups $n$ and the number of bullets $\mathrm{m}$ from zero to infinity, the change trend of $\delta_{\hat{R}_{50}}$ into three-dimensional images:

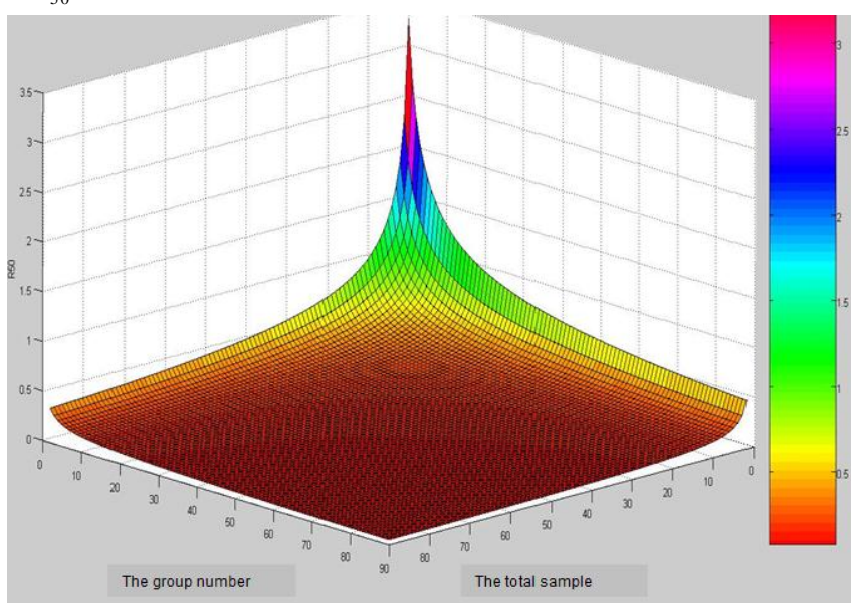

Fig. 3. $\delta_{\hat{R}_{50}}$ and the group $\mathrm{n}$ and group $\mathrm{M}$ relative number.

Seen from Fig. 3, under certain shooting sample condition, the bullet firing dispersion estimation accuracy of $\delta_{\hat{R}_{50}}$ was decided by the group number $n$ and bullet number $m$, the firing precision of the $\delta_{\hat{R}_{50}}$ conditions, the optimal number must have a group of $n$ and the bullets of $m$ in pairs.

\section{Shooting Groups and Bullets to Determine the Number}

This paper will analysis and deduction on the basis of the above theory, the test data for the support, to confirm the bullet dispersion test sample.

Check our troops in recent years a small-bore rifle bullet dispersion test results, the numerical values are listed in the following table:

For a small-bore rifle bullet. $\left(R_{50}\right)_{0}$ in one hundred meters is $2.2 \mathrm{~cm}$, in three hundred meters is $6.6 \mathrm{~cm}$.

TABLE I: A SMALL-BORE RIFLE BULLET DisPERSION TEST FOR 100M

\begin{tabular}{ccccc}
\hline & 2005 & 2004 & 2002 & 1999 \\
\hline 1 & 2.2 & 1.8 & 2.2 & 1.3 \\
\hline 2 & 1.9 & 2.1 & 2.7 & 1.8 \\
\hline 3 & 2.3 & 1.6 & 1.9 & 1.9 \\
\hline 4 & 1.7 & 1.9 & 2.3 & 1.4 \\
\hline 5 & 2.1 & 2.1 & 2.2 & 1.2 \\
\hline 6 & 2.1 & 1.7 & 2.2 & 1.1 \\
\hline
\end{tabular}


Check for years archive in our troops, during the period $1999 \sim 2005$,some small-bore rifle bullets in the dispersion test $\left(R_{50}\right)_{0} 0$ for $100 \mathrm{~m}, 300 \mathrm{~m}$ results are shown in Table I and Table II.

TABLE II: A SMALL-BORE RIFLE BULLET DISPERSION TEST FOR 300M

\begin{tabular}{ccccc}
\hline & 2005 & 2004 & 2002 & 2000 \\
\hline 1 & 6.2 & 8.7 & 8.1 & 5.2 \\
\hline 2 & 7.0 & 5.8 & 5.1 & 4.9 \\
\hline 3 & 6.2 & 8.9 & 6.0 & 4.5 \\
\hline 4 & 6.5 & 6.1 & 4.6 & 4.2 \\
\hline 5 & 7.0 & 5.4 & 5.8 & 5.2 \\
\hline 6 & 4.5 & 6.5 & 5.6 & 5.4 \\
\hline
\end{tabular}

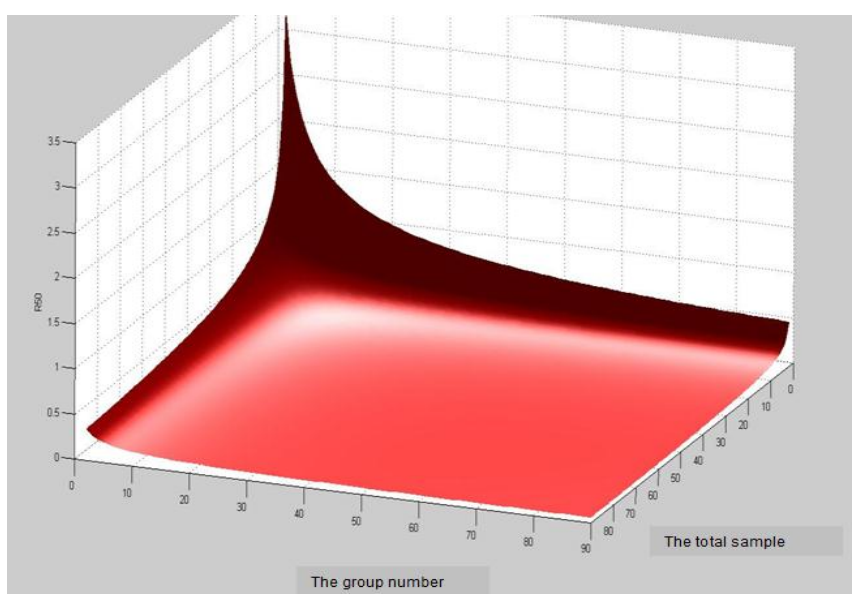

Fig. 4. The relational graph between estimated accuracy and group number the bullet number in a small-bore rifle bullet dispersion test for $100 \mathrm{~m}$.

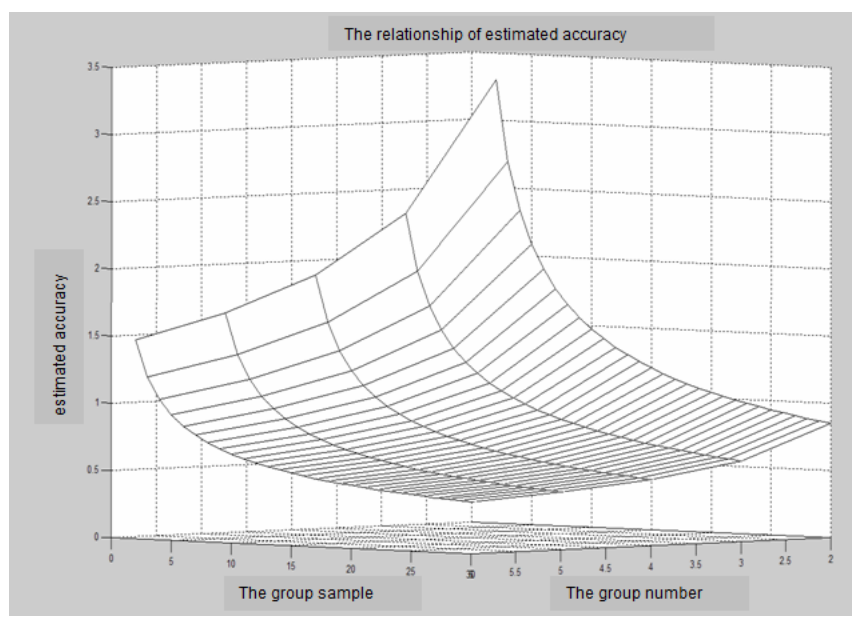

Fig. 5. Fig. 4 amplification.

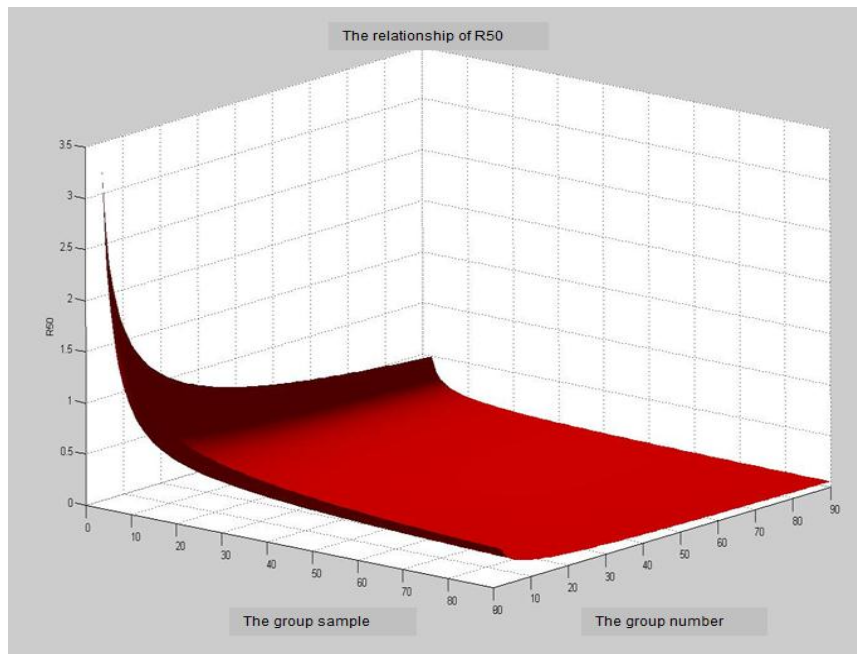

Fig. 6. The relational graph between estimated accuracy and group number the bullet number in a small-bore rifle bullet dispersion test for $300 \mathrm{~m}$.

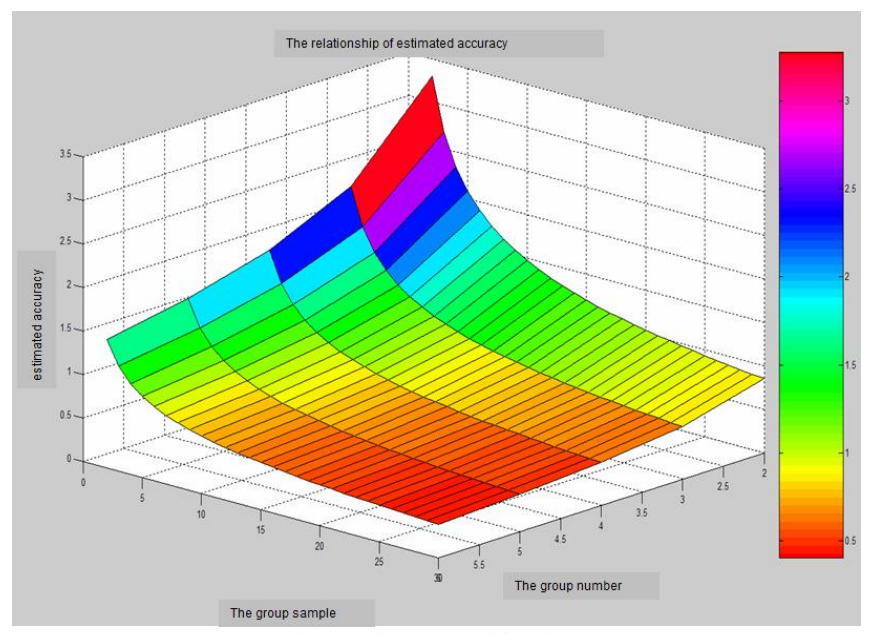

Fig. 7. Fig. 6 amplification.

According to the above formula (10)-(12), and the total sample size of 40 bullets, transform test group number $n$ and bullet number $\mathrm{m}$, obtains the $100 \mathrm{~m}$ dispersion test in the estimation accuracy and the group number, the bullet number of the relationship between 3D graph ( Fig. 4-Fig. 7).

From Fig. 5 and Fig. 7 can be seen, the estimation accuracy significantly change state (i.e. color changes range) corresponding to 4 groups, each group in group ten bullets.

\section{CONCLUSION}

From the above analysis results show that, for small-bore rifle bullet, the best number of bullet samples and packet for dispersion test should be: overall sample size of 40 bullets, 4 groups, each group of 10 rounds.: total number of samples taken 40 bullets, divided into 4 groups, each group has 10 bullet. Using the method described in this article, can be based on extensive experimental dispersion data, scientific and intensive sample take the bullet test.

\section{REFERENCES}

[1] X. Tang et al., Test Analysis and Evaluation of Weapon Equipment in Small Samples, National Defense Industry Press, China, 2001, pp. 56-112.

[2] Z. Yan et al., Statistical Analysis of Test Data, National Defense Industry Press, China, 2000, pp. 185-220.

[3] C. Peng, Error and Return, Weapon Industry Press, China, 1989, pp. $1-11$.

[4] Z. He and G. Li, Probability Theory and Mathematical Statistics, Weapon Industry Press, China, 1993, pp. 189-230.

[5] C. Pan and Z. Han, Weapons and Ammunition Test and Inspection Probability, Weapon Industry Press, China, 1980, pp. 153-178.

[6] G. Wang et al., "Dispersion of multiple weapon system simulation technology," Journal of System Simulation, vol. 16, pp. 963-966, May, 2004.

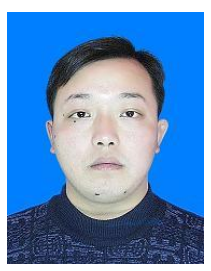

Xu Youliang was born in 1978, Sichuan province, China, who received his master degree from University of Science and Technology of China. He is a mechanical and electronic professional, and a senior engineer of Baicheng Ordnance Test Center. His present professional occupation is focused on mechanical performance test. He has many years of experience in simulation testing and data processing, His current research interests are in the comprehensive evaluation of mechanical properties. 


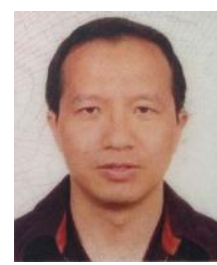

Zhang Junbin was born in 1970, Sichuan province, China. He received his bachelor degree from North China Institute of Technology. He is a mechanical and electronic professional, and a senior engineer of Baicheng Ordnance Test Center. He present professional occupation is focused on mechanical design and analysis of optimization. At present, his research interest is data optimization analysis.

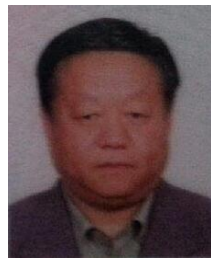

Ma Li was born in 1962, Jilin province, China. He received his master degree from University of Science and Technology of China. He is a mechanical and electronic professional, a researcher of Baicheng Ordnance Test Center and a chief engineer. His present professional occupation is focused on large overall data analysis work. His current research interest is in the mechanical system overall planning.

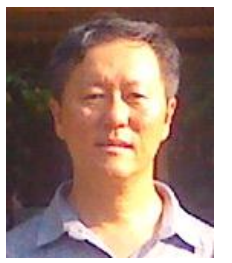

Shan Yonghai was born in 1964, Jilin province, China. He is a researcher of Baicheng Ordnance Test Center, a PhD of Xian Jiao Tong University, and a systems engineering professional. His present professional occupation is focused on mechanical design and test appraisal work. His current research interests is in the mechanical system overall planning. 